\title{
User requirements for wearable smart textiles. Does the usage context matter (medical vs. sports)?
}

\author{
Julia van Heek ${ }^{1}$, Anne Kathrin Schaar ${ }^{1}$, Bianka Trevisan ${ }^{2}$, Patrycja Bosowski ${ }^{3}$, Martina Ziefle ${ }^{1}$ \\ ${ }^{1}$ Communication Science, RWTH Aachen University \\ ${ }^{2}$ Textlinguistics, RWTH, Aachen University \\ ${ }^{3}$ Institut für Textiltechnik, RWTH Aachen University
}

\begin{abstract}
Wearable smart textiles are a promising approach to provide health related services (e.g., permanent monitoring of vital parameters), and can be used in different context (such as sports or health care). But so far, smart textiles [ST] have not significantly penetrated the market. To identify possible reasons, the acceptance of important stakeholders must be investigated. This paper offers a first step in this direction by presenting a questionnaire study on user requirements of smart wearable textiles. To find out whether there is a generic attitude towards ST, this study is focused on a comparison of user requirements in two settings, sport and health care, as two central fields of application for this technology.

First results show that there are differences in the evaluation of requirements of ST pertaining to ease of use, permanent information provision about one's health status, data security as well as the design of such textiles, based on the usage context.
\end{abstract}

\section{Keywords}

User requirements, technology acceptance, user diversity, smart textiles, medical technology

\section{INTRODUCTION}

Innovations in the field of medical technology like wearable ST are promising approaches to support old and frail people in the future, but also offer prevention and protection during sports and training. Especially in times of demographic changes, such solutions could be beneficial for the provision of adequate and dignified health care. But do these approaches also match users' needs and do these needs differ depending on the field of application? Due to the fact that a lot of inventions in the sector of medical technology fail their establishment in the market, it is essential to learn more about the reasons for this failure. A lack of acceptance amongst stakeholders (e.g., potential end-users) could be one reason. As there is only little knowledge about the stakeholders' attitude towards wearable ST, an interdisciplinary team of social scientist and textile engineers initiated a cooperation at the RWTH Aachen University in Germany to investigate acceptance parameters in the context of wearable ST. The presented research is a result of this cooperation, which is in a first step focused on end-user requirements in two usage scenarios (i.e., medical and sports) supposed to be likely fields of application.

Permission to make digital or hard copies of all or part of this work for personal or classroom use is granted without fee provided that copies are not made or distributed for profit or commercial advantage and that copies bear this notice and the full citation on the first page. To copy otherwise, to republish, to post on servers or to redistribute to lists, requires prior specific permission and/or a fee.

User Centered Design 2014, May 20-22

Copyright (C) 2014 ICST 978-1-63190-011-2

DOI 10.4108/icst.pervasivehealth.2014.255179

\subsection{Smart textiles}

The term smart textiles stands for a huge field of different textiles and materials, defined as intelligent materials and systems, that are able to sense and/or interact with their environment in a predictable and useful way [13]. There are generally three functions ST can take: a) sensing, b) actuating, and c) adapting.

With these abilities, ST are interesting for different fields of application. One example is the health care sector [14][18] in which ST approaches are often focused on health monitoring. The integration of sensors into shirts [6] and other objects worn on a person's body (e.g., belt [12], shoes [5], jewelry [1]) allow for nonstop checking of vital parameters like temperature or pulse rate. This vision of easy and continuous monitoring would be a great support for medical care within the home environment. That is especially relevant for old and/or frail people that are not hospitalized. In the focus of the presented research, a concept of a smart shirt for vital parameter measurement is introduced in line with actual research in the field of smart shirts [14][8][6][2].

\subsection{Technology acceptance - of new medical technologies}

The research field of medical technology acceptance is based on results of general technology acceptance research [3][16]. The key representatives for these theories are the technology acceptance model (TAM) of Davis [4] and its further development the unified theory of acceptance and use of technology (UTAUT) by Venkatesh et al. Two central elements of both theories are the influence of perceived ease of use and perceived usefulness on the attitude towards using technology and the actual system use. Whereas TAM was first focused on few moderating aspects in the context of acceptance, the further development of both approaches integrated more aspects such as voluntariness of use, output quality, subjective norms [17], or social influence [16]. As these models revealed a high level of prediction for the acceptance of information and communication technologies, they were also used to investigate other forms of technology, e.g., medical technology. Results of medical technology acceptance research revealed that ease of use and usefulness are important, as are other aspects like privacy [7][21], trust [9], and security [19][20]. In the context of acceptance research of ST, first results revealed that acceptance or attitude towards this technology depends on aspects of user diversity [15]. Furthermore, it could be shown that enhancing control, safety, and mobility for patients are benefits of ST, whereas technical errors, obstructions in everyday life as well as high costs could be identified as barriers in the context of wearable ST [11].

\section{MAIN FOCUS OF THE STUDY AND QUESTIONS ADDRESSED}

In order to find out whether the usage context influences the acceptance of wearable ST and whether factors of user diversity 
impact this, the following two research questions were focused upon:

RQ1: Is there a difference in the evaluation of user requirements between medical and sports usage scenarios?

RQ2: Do user diversity factors (i.e., age, gender, prior knowledge about ST, practical experience with ST, and health status) influence the evaluation of acceptance parameters?

\section{METHOD}

To reach a large number of participants, the questionnaire method was chosen. Data sets were collected in an online survey conducted in Germany between July and August 2012. Participants were asked via email to take part in the study and were given a link to the questionnaire. Completing the questionnaire took about 20 minutes.

\subsection{Variables}

The independent variables were age, gender, health status, prior knowledge about ST, and practical experience with ST. We selected the evaluation of user requirement factors for ST usage (see Section 3.2) as dependent variables. The requirement factors were chosen based on a prior study of the consortium which was focused on a qualitative and explorative evaluation of user requirements of smart wearable textiles for sports and medical purposes [15].

\subsection{The questionnaire instrument}

The questionnaire was structured as follows: the first part assessed demographic characteristics such as age, gender, dealing with health, sports activity, chronic disease, interest in technology, and technology expertise (ownership, frequency of use, perceived usefulness of technical devices). The second part asked for prior knowledge and current practical experiences with ST. The third part assessed the participants' ST acceptance in a medical and in a sport usage scenario. In both scenarios, 13 different requirements were presented: warning function, functionality \& reliability, fashionable look, discreet vs. striking design, wearing comfort, exclusion of physical dangers, transmission \& storage of data, permanent information about health status, easy cleaning, durability, price, ease of use, data security.

\section{RESULTS}

This section presents the results pertaining to the research questions. Data were analyzed by descriptive analysis as well as Spearman's correlation. Additionally, one-way repeated measure ANOVA tests were used. The level of significance was set at alpha $=0.05$.

\subsection{Sample}

In total, 172 participants between 15 and 75 years of age took part in the survey. The mean age of the participants was $30.9(\mathrm{SD}=$ 10.29). $55.2 \%$ of the respondents $(\mathrm{N}=95)$ were men and $44.8 \%$ women $(\mathrm{N}=77)$. In addition to general demographic data, all participants were asked about their physical activity. The majority of $75.6 \%$ reported to do sports, while $24.4 \%$ indicated not to be active. Regarding their health status, $19.2 \%$ stated to suffer from a chronic disease, the rest $(80.8 \%)$ claimed to be healthy. Concerning current knowledge about ST, the majority (73.3\%) indicated to have prior knowledge while $26.7 \%$ quoted to have no prior knowledge. Furthermore, $10.5 \%$ stated that they already had practical experience with ST, but the large majority of $89.5 \%$ did not have any practical experience with ST so far.
For our analysis, the following group classifications have been chosen: the participants were differentiated by age, gender, chronic disease (yes/no), prior knowledge about ST (yes/no), and practical experience with ST (yes/no). To study age effects, a between factor "age groups" was created with three sub-groups: "young adults in education" (aged 15-25 years), "young professionals" (aged 26-40 years), and "older professionals and pensioners" (aged 41-75 years).

\subsection{Comparing medical and sports scenarios}

In this section, the results concerning user requirements for ST are presented. Analyses were focused on identifying differences between the two usage-scenarios in this study (medical vs. sports).

\subsubsection{Descriptive analysis}

Based on descriptive analysis, we could reveal a general tendency for confirming the presented requirements. All questions were answered by the whole sample $(\mathrm{N}=172)$ : The three most important requirements in the medical usage scenario were: exclusion of physical dangers $\left(\mathrm{M}_{\mathrm{med}}=5.65 ; \mathrm{M}_{\text {sport }}=5.60\right)$, functionality \& reliability $\left(\mathrm{M}_{\mathrm{med}}=5.56 ; \mathrm{M}_{\mathrm{sport}}=5.55\right)$, and high wearing comfort $\left(\mathrm{M}_{\text {med }}=5.38 ; \mathrm{M}_{\text {sport }}=5.15\right)$ (see Figure 1). The first two items are the same for the sports related usage scenario whereas the third most important item here was easy cleaning $\left(\mathrm{M}_{\text {med }}=5.22 ; \mathrm{M}_{\text {sport }}=\right.$ 5.26). In comparison, the requirements price $\left(\mathrm{M}_{\text {med }}=3.55 ; \mathrm{M}_{\text {sport }}=\right.$ $3.64)$ and discreet \& striking design $\left(\mathrm{M}_{\text {med }}=4.38 ; \mathrm{M}_{\text {sport }}=3.32\right)$ play only minor roles in explaining the acceptance of ST.

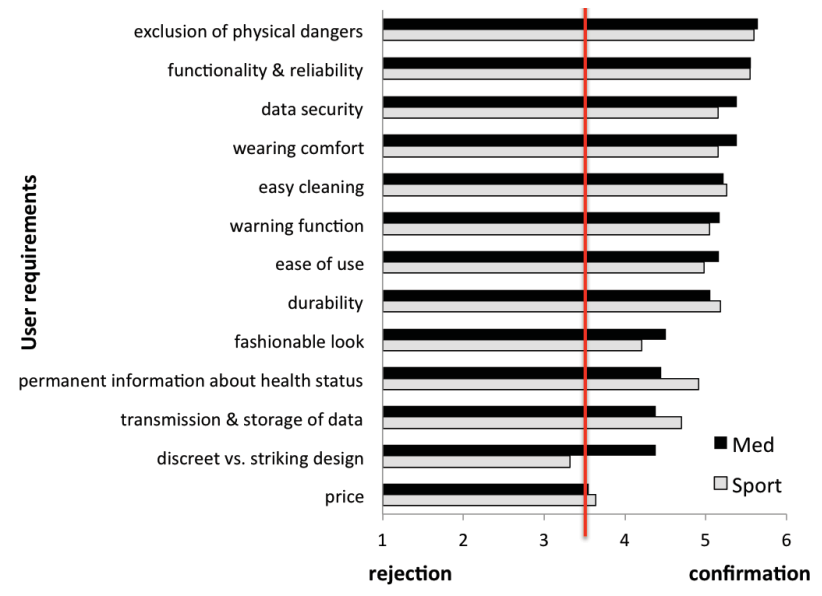

Figure 1: Mean comparison $(\mathrm{N}=172)$ of user requirements in sports and medical scenarios on a six-point Likert scale $(1=$ total rejection, $6=$ total confirmation $)$

\subsubsection{Correlation analysis}

Spearman correlations between the different aspects of user requirements revealed highly significant correlations between the evaluations in the two scenarios, except for the factor discreet design (see Table 1).

In summary, we can say that almost all items were evaluated to be relevant in both settings. We could also reveal that items related to security and reliability are most important whereas aspects like a fashionable look, data transmission, and price play minor roles. Additionally, a high correlation between the evaluations of user requirement factors could be observed. Based on these results, we hypothesize that there is a generic attitude towards ST no matter what usage context. 
Table 1. Results for Spearman correlation analysis for user requirements in the two usage scenarios medical vs. sport

\begin{tabular}{|l|c|c|}
\hline \multicolumn{1}{|c|}{ item } & Spearmans-Rho & p \\
\hline fashionable look & 0.440 & 0.000 \\
\hline transmission \& storage of data & 0.535 & 0.000 \\
\hline easy cleaning & 0.449 & 0.000 \\
\hline permanent information about health status & 0.343 & 0.000 \\
\hline data security & 0.645 & 0.000 \\
\hline functionality \& reliability & 0.387 & 0.000 \\
\hline durability & 0.552 & 0.000 \\
\hline wearing comfort & 0.374 & 0.000 \\
\hline exclusion of physical dangers & 0.549 & 0.000 \\
\hline ease of use & 0.514 & 0.000 \\
\hline discreet vs. striking design & 0.019 & 0.805 \\
\hline price & 0.685 & 0.000 \\
\hline warning function & 0.588 & 0.000 \\
\hline
\end{tabular}

To find out whether these assumptions are conclusive, the next section presents the results of one-way repeated measure analysis with the user diversity factors age, gender, prior knowledge about ST, practical experience with ST, and health status as betweensubject factors.

\subsubsection{Comparing the scenarios with repeated}

measure analysis

In contrast to our hypothesis, repeated measures analysis revealed significant differences between the medical and the sports scenario for six of the 13 requirements (see Table 2).

Table 2. Significant results for repeated measure analysis between user requirements in a medical and a sports scenario

\begin{tabular}{|l|c|c|c|c|}
\hline \multicolumn{1}{|c|}{$\begin{array}{c}\text { Items for user } \\
\text { requirements }\end{array}$} & $\begin{array}{c}\text { Wilks' } \\
\text { Lambda }\end{array}$ & $\mathbf{F ( 1 , 1 7 1 )}$ & $\mathbf{p}$ & $\boldsymbol{\eta}^{\mathbf{2}}$ \\
\hline data security & .95 & 10.02 & $<0.005$ & .055 \\
\hline ease of use & .96 & 7.16 & $<0.01$ & .040 \\
\hline fashionable look & .93 & 12.21 & $<0.005$ & .067 \\
\hline $\begin{array}{l}\text { permanent information } \\
\text { about health status }\end{array}$ & .88 & 24.40 & $<0.001$ & .125 \\
\hline $\begin{array}{l}\text { transmission \& storage } \\
\text { of data }\end{array}$ & .93 & 13.34 & $<0.001$ & .072 \\
\hline design & .93 & 154.51 & $<0.001$ & .475 \\
\hline
\end{tabular}

Based on Pierce [10], we could reveal small effects within our sample for the factors data security and ease of use; medium effects for fashionable look, transmission and storage of data, and permanent information about health status; and a large effect was found for design. To determine whether user factors, i.e., age, gender, prior knowledge about ST, practical experience with ST, and health status have any influence on these aspects, the next section presents results of repeated measure analyses with diversity factors as between-subject factors.

\subsubsection{Do factors of user diversity make a difference?}

According to our research questions, we integrated diversity factors as between-subject factors into the analysis. As the upper part of Table 3 illustrates, we found significant influences for the diversity factors partial experience with ST, prior knowledge, and age on items that revealed a significant difference in the prior repeated measure analysis (see Section 4.2.3). For ease of use and design, we could also reveal a significant difference in the evaluation under consideration of practical experience. For transmission \& storage of data we found a significant influence of the factor prior knowledge about ST. Additionally, we could reveal significant differences in the evaluation of the factor ease of use under consideration of the diversity factor age. For the requirements that revealed no significant differences between both scenarios (see Section 4.2.3), we could reveal differences for: functionality \& reliability under consideration of the factor age; and durability under consideration of health status as well as prior knowledge about ST.

Table 3. Results for repeated measure analysis between user requirements in medical and sports scenarios in consideration of user diversity factors as between-subject factors

\begin{tabular}{|l|l|c|c|c|c|}
\hline \multicolumn{1}{|c|}{$\begin{array}{c}\text { diversity } \\
\text { factor }\end{array}$} & $\begin{array}{l}\text { user } \\
\text { requirements }\end{array}$ & $\begin{array}{c}\text { Wilks' } \\
\text { Lambda }\end{array}$ & $\begin{array}{c}\mathbf{F} \\
\mathbf{( 1 , 1 7 0 )}\end{array}$ & $\mathbf{p}$ & $\boldsymbol{\eta}^{2}$ \\
\hline $\begin{array}{l}\text { practical } \\
\text { experience }\end{array}$ & ease of use & .97 & 11.68 & $<0.01$ & .064 \\
\cline { 2 - 6 } $\begin{array}{l}\text { prior } \\
\text { knowledge }\end{array}$ & $\begin{array}{l}\text { transmission } \\
\& \\
\text { storage of } \\
\text { data }\end{array}$ & .98 & 3.69 & $=.000$ & .021 \\
\hline age & ease of use & .97 & 2.99 & $=.000$ & .021 \\
\cline { 2 - 6 } & $\begin{array}{l}\text { functionality } \\
\&\end{array}$ & $=97$ & .17 & $=.000$ & .032 \\
\hline reliability & & & & .034 \\
\hline $\begin{array}{l}\text { health } \\
\text { status }\end{array}$ & durability & .97 & 5.69 & $=.000$ & .032 \\
\hline $\begin{array}{l}\text { prior } \\
\text { knowledge }\end{array}$ & durability & .98 & 2.99 & $=.000$ & .017 \\
\hline
\end{tabular}

In summary of this section, we can say there is no generic attitude towards requirement of ST. User diversity factors like practical experience and prior knowledge with/about ST as well as health status and age also play roles in the evaluation within different usage settings.

\section{DISCUSSION, LIMITATIONS, AND FUTURE RESEARCH}

The presented research was part of an interdisciplinary cooperation, initiated to find out which factors influence the acceptance of smart wearable textiles in different future usage settings. In this context, a first look on future users, one group of possible, relevant stakeholders, revealed that there is no generic attitude towards ST when comparing requirements for a sports related setting to that of a health care setting as the two central and possible future fields of application.

When it comes to medical use, aspects like data security, ease of use, fashionable look, and design are of high importance. The aspects permanent information about the health status and data transmission are more important for sportive usage purposes.

Regarding the question whether user diversity makes a difference in the evaluation, we could reveal that especially prior knowledge 
and experience with ST lead to a higher level of acceptance. Based on our findings, we can say that a tailored introduction and design of ST are necessary to introduce ST successfully. When it comes to the use in health care, it is important that three aspects are given: ease of use, security as well as a fashionable look. In this context, usage is impaired by illness and therefore involuntary use. Accordingly, it is even more important that patients feel good with these technologies in their everyday life (fashionable look), that they can rely on them completely (security), and that they are able to use them easily to get the feeling of mastering its control (ease of use). For ST usage in sports, we find different conditions. Here, usage is absolutely voluntary, which leads to other challenges: Our sample revealed that especially high-end functionalities (transmission of data and permanent information about health status) are of importance. Therefore, future studies should focus on the integration of more specific target groups (patients with diseases that could benefit from health monitoring via wearable ST as well as sportsmen and women). Additionally, hands-on-research with prototypes would help overcome the hypothetical status and reveal more solid data, especially as experience with technology is a key parameter for acceptance. Furthermore, the role of other stakeholders in the context of ST such as industry and professional users (e.g., nursing staff, physicians, or hospital management) as well as research promotion should be investigated to learn more about interests and attitudes behind the scenes.

For potential users we can say that the usage context does matter and that a user centered design and provision information is the key for more acceptance in the context of ST, as a promising field for different purposes in the future.

\section{REFERENCES}

[1] Asada, H.H. et al. 2003. Mobile monitoring with wearable photoplethysmographic biosensors. Engineering in Medicine and Biology Magazine, IEEE. 22, 3 (2003), 2840.

[2] Catrysse, M. et al. 2004. Towards the integration of textile sensors in a wireless monitoring suit. Sensors and Actuators A: Physical. 114, 2-3 (Sep. 2004), 302-311.

[3] Davis, F.D. 1985. A technology acceptance model for empirically testing new end-user information systems: theory and results. John C. Henderson., Sloan School of Management., Sloan School of Management.

[4] Davis, F. et al. 1989. User Acceptance Of Computer Technology: A Comparison Of Two Theoretical Models. Management science. 35(8), (1989), 928ff.

[5] Jagos, H. et al. 2007. Erste Schritte bei der Entwicklung instrumentierter Schuhe zur Sturzvorbeugung alter Menschen. (Linz, Austria, Sep. 2007).

[6] Kim, S. et al. 2008. Influence of contact pressure and moisture on the signal quality of a newly developed textile ECG sensor shirt. 5th International Summer School and Symposium on Medical Devices and Biosensors, 2008. ISSS-MDBS 2008 (Jun. 2008), 256-259.

[7] Lahlou, S. et al. 2005. Privacy and trust issues with invisible computers. Commun. ACM. 48, 3 (2005), 59-60.

[8] Lee, Y.-D. and Chung, W.-Y. 2009. Wireless sensor network based wearable smart shirt for ubiquitous health and activity monitoring. Sensors and Actuators B: Chemical. 140, 2 (Jul. 2009), 390-395.

[9] Luo, X. et al. Examining multi-dimensional trust and multi-faceted risk in initial acceptance of emerging technologies: An empirical study of mobile banking services. Decision Support Systems. 49, 2, 222-234.

[10] Pierce, C.A. et al. 2004. Cautionary Note on Reporting EtaSquared Values from Multifactor ANOVA Designs. Educational and Psychological Measurement. 64, 6 (Jan. 2004), 916-924.

[11] Schaar, A.K. and Ziefle, M. 2011. Smart clothing: Perceived benefits vs. perceived fears. 2011 5th International Conference on Pervasive Computing Technologies for Health care (PervasiveHealth) (May 2011), 601-608.

[12] Scheermesser, M. et al. 2008. User acceptance of pervasive computing in health care: Main findings of two case studies. Pervasive Computing Technologies for Health care, 2008. PervasiveHealth 2008. Second International Conference on Pervasive Computing Technologies for Health care (2008), 205-213.

[13] Schwarz, A. et al. 2010. A roadmap on smart textiles. Textile Progress. 42, 2 (Jul. 2010), 99-180.

[14] Sungmee Park and Jayaraman, S. 2003. Enhancing the quality of life through wearable technology. IEEE Engineering in Medicine and Biology Magazine. 22, 3 (May 2003), 41-48.

[15] Trevisan, B. et al. 2012. Acceptability Studies on Smart Texiles: An Interdisciplinary Cooperation to Understand Users' Acceptance. Extended Abstract at Fiber Society Spring Conference 2012. (St. Gallen (CH), 25.5 2012).

[16] Venkatesh, V. et al. 2003. User Acceptance of Information Technology: Toward a Unified View. MIS Quarterly. 27, 3 (2003), 425-478.

[17] Venkatesh, V. and Davis, F.D. 2000. A theoretical extension of the technology acceptance model: Four longitudinal field studies. Management science. 46, 2 (2000), 186-204.

[18] Ziefle, M. and Röcker, C. 2010. Acceptance of Pervasive Health care Systems: A comparison of different implementation concepts. 4th ICST Conference on Pervasive Computing Technologies for Health care 2010 (2010).

[19] Ziefle, M. and Schaar, A.K. 2011. Gender differences in acceptance and attitudes towards an invasive medical stent. Electronic Journal of Health Informatics. 6, 2 (2011), 118.

[20] Ziefle, M. and Schaar, A.K. 2010. Technical Expertise and Its Influence on the Acceptance of Future Medical Technologies: What Is Influencing What to Which Extent? HCI in Work and Learning, Life and Leisure. G. Leitner et al., eds. Springer Berlin Heidelberg. 513-529.

[21] Ziefle, M. and Wilkowska, W. 2010. Technology acceptability for medical assistance. Proceedings of 4th ICST Conference on Pervasive Computing Technologies for Health care 2010 (2010), 1-9. 
